\title{
THREE-DIMENSIONAL SIMULATION STUDY OF PLASMOID INJECTION INTO MAGNETIZED PLASMA
}

\author{
Y. SUZUKI, T.-H. WATANABE, A. KAGEYAMA, T. SATO AND \\ T. HAYASHI \\ Theory and computer simulation center, \\ National Institute for Fusion Science \\ Toki 509-52, Japan
}

\section{Introduction}

Resent observations suggest that, during solar flares, plasmoids are injected into the interplanetary medium (Stewart et al., 1982). It has also been pointed out that solar wind irregularities modeled as plasmoids are penetrated into the magnetosphere (Lemaire, 1977). These plasmoid injections are considered to be an important process because they transfer mass, momentum, and energy into such magnetized plasma regions. Our objective is to investigate the dynamics of a plasmoid, which is injected into a magnetized plasma region and to reveal mechanisms to transfer them. To achieve this, we carried out three-dimensional magnetohydrodynamic (MHD) simulations.

\section{3D Dynamics of Injected Plasmoid}

The simulation model is the same as that in Suzuki et al. (1997). The simulation region is composed of two cylinders which connect with each other. One with a smaller radius corresponds to a injection region in which the plasmoid is initially located, and the other with a larger radius is a magnetized plasma region where the magnetic field is almost unidirectional and perpendicular to the injection direction. The plasmoid is accelerated in the injection region into the magnetized plasma region.

The simulation result is shown in Figure 1. We can see that at $t=20$ the plasmoid enters the magnetized plasma region, suffering from the tilting instability. Also, at this time, the plasmoid field reconnects with the field 


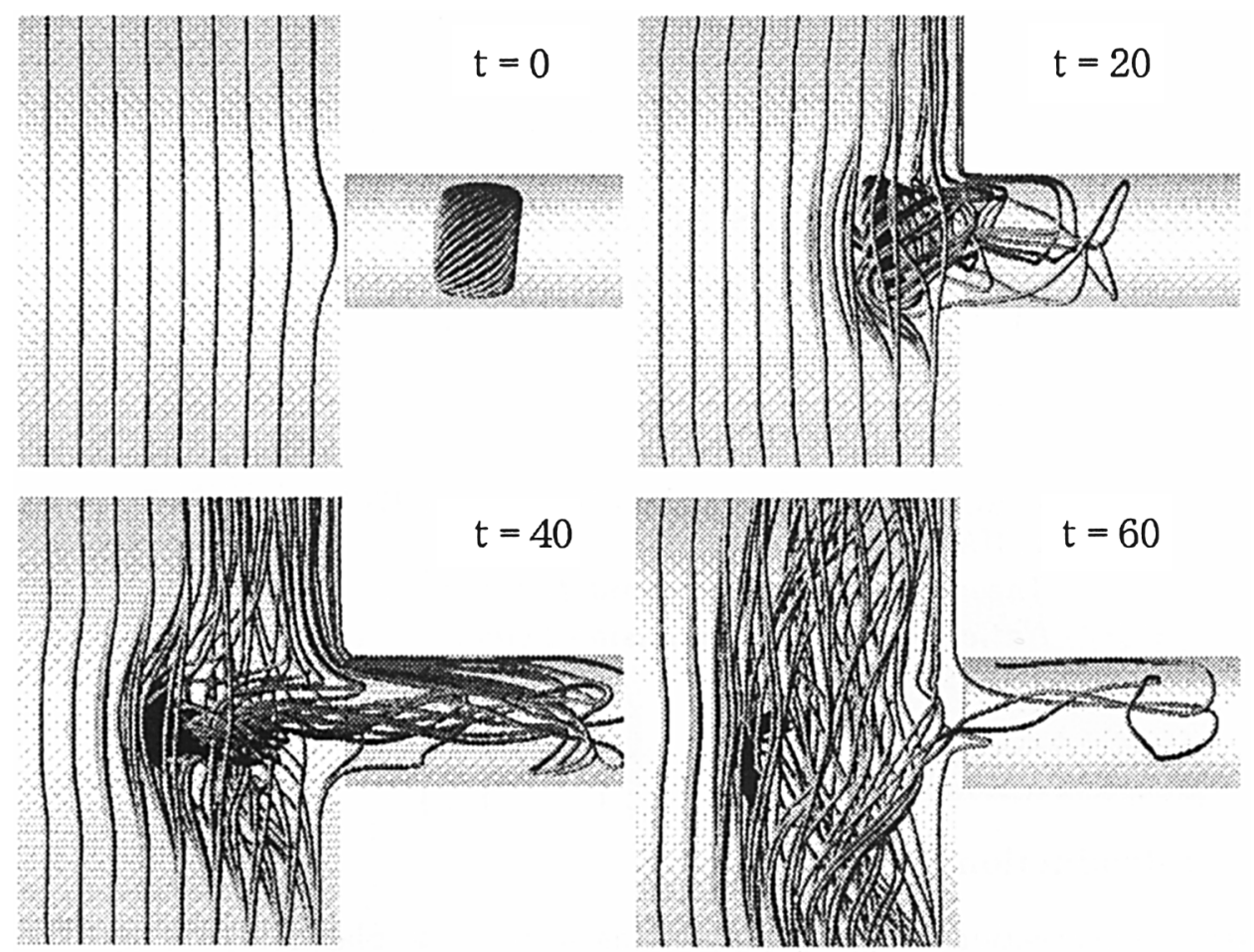

Figure 1. The structure of the magnetic field lines and the iso-value surface of the high density plasma (black region) at $t=0,20,40,60$. The time is normalized by the characteristic Alfvén transit time.

in the magnetized plasma region. As time goes, the magnetic reconnection process successively proceeds $(t=40)$. Finally the magnetic configuration of the plasmoid is disrupted $(t=60)$. As a result, the high density plasma confined in the plasmoid is supplied in the magnetized plasma region. From these results we can see that the plasmoid injection is an important process especially to transfer the plasmoid mass into the magnetized plasma region, where the magnetic reconnection is a key mechanism.

\section{References}

Stewart, R.T., Dulk, G.A., Sheridan, K.V., House, L.L., Wanger, W.J., Sawyer, C.J., and Illing, R., (1982) Visible Light Observations of a Dense Plasmoid Associated with a Moving Type IV Solar Radio Burst, Astron. Astrophys., 116., pp. 217-223.

Lemaire, J., (1977) Impulsive Penetration of a Filamentary Plasma Element into the Magnetosphere of the Earth and Jupiter, Planet. Space Sci., 25., pp. 887-890.

Suzuki, Y., Watanabe, T.-H., Kageyama, A., Sato, T., and Hayashi, T. (1997) to be published in Proc. of the Joint Conference of 11th International Stellarator Conference 8 8th International Toki Conference on Plasma Physics and Controlled Nuclear Fusion, ed. Y.Ueda JPFR Series 1. 